psychopraxis. neuropraxis $2021 \cdot 24: 348-355$ https://doi.org/10.1007/s00739-021-00758-5 Angenommen: 27. September 2021 Online publiziert: 26 . Oktober 2021

๑ Der/die Autor(en) 2021

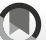

Simon Fandler-Höfler · Markus Kneihsl · Thomas Gattringer

Universitätsklinik für Neurologie, Medizinische Universität Graz, Graz, Österreich

\title{
Update zur Sekundärprophylaxe nach ischämischem Schlaganfall
}

nach Schlaganfall vielfach nicht adäquat kontrolliert/eingestellt.

20-25.000 Menschen einen ischämischen Schlaganfall, ca. $20 \%$ davon machen Rezidivschlaganfälle aus. Die Sekundärprophylaxe nach einem ischämischen Schlaganfall hat eine zentrale Bedeutung, da rezidivierende zerebrovaskuläre Ereignisse, aber auch kardiovaskuläre Folgeerkrankungen bei Schlaganfallpatient ${ }^{*}$ innen häufig sind und mit einer deutlich erhöhten Morbidität und Mortalität einhergehen. Das Rezidivrisiko nach erlittenem ischämischem Schlaganfall ist mit etwa $20 \%$ innerhalb von 5 Jahren erheblich und besonders in den ersten Monaten nach dem Ereignis deutlich erhöht. Der überwiegende globale Anteil der Schlaganfälle lässt sich durch Kontrolle/adäquate Einstellung von modifizierbaren vaskulären Risikofaktoren (Bluthochdruck, ungesunde Ernährung, Bewegungsmangel, abdominelle Adipositas und Rauchen) um mindestens $80 \%$ reduzieren (InterstrokeStudie, bestätigt durch Global Burden of Disease Report).

Vor allem die kombinierte Therapie vaskulärer Erkrankungen/Risikofaktoren scheint speziell in der Sekundärprävention nach Schlaganfall einen besonderen additiven Effekt $\mathrm{zu}$ haben (Medikamente wie ASS, Statine, Antihypertensiva; kombiniert mit mediterraner, salzarmer Diät und Bewegung, Raucherstopp, kein übermäßiger Alkoholkonsum). Ein allgemein akzeptiertes Blutdruckziel nach Schlaganfall/TIA beträgt $<130 / 80 \mathrm{~mm} \mathrm{Hg}$ für die meisten Patient*innen, wobei es keine Präferenz hinsichtlich einer antihypertensiven Substanzklasse gibt. Leider sind der Lebensstil/die vaskulären Risikofaktoren

Literatur bei den Verfassern.

\section{》) Die Sekundärprophylaxe ist auf die jeweilige Schlaganfallä- tiologie ausgerichtet}

Basierend auf wissenschaftlichen Erkenntnissen der letzten Jahre setzt sich der Trend $\mathrm{zu}$ einer individualisierten Schlaganfallnachsorge und damit $\mathrm{zu}$ einer auf die Schlaganfallätiologie ausgerichteten Sekundärprophylaxe fort. Der Erhebung der konkreten Schlaganfallätiologie kommt damit besonders große Relevanz zu. • Abb. 1 zeigt schematisch die häufigsten Schlaganfallätiologien und deren Grundsätze in der Sekundärprophylaxe.

\section{Generelle medikamentöse Therapiestrategien}

\section{Thrombozytenaggregations- hemmung}

Die Acetylsalicylsäure (ASS) hat ihren Stellenwert als Basistherapie in der Sekundärprophylaxe des nicht-kardioembolischen Schlaganfalls nicht verloren. Da das Rezidivrisiko eines ischämischen Schlaganfalls allerdings in den ersten Tagen bis Wochen nach dem Ereignis am höchsten ist und mit der Zeit abnimmt, haben rezente Studien intensivierte Sekundärprophylaxeschemata in den ersten Monaten untersucht. Drei randomisiert-kontrollierte Studien der letzten Jahre (CHANCE, POINT und THALES) konnten zeigen, dass eine vorübergehende duale Thrombozytenaggregationshemmung in den ersten 1-3 Monaten nach nicht-kardioem- bolischem leichten Schlaganfall oder Hochrisiko-TIA zu einer deutlichen Reduktion an ischämischen Rezidivereignissen führt. Gleichzeitig zeigte sich in Zusammenschau der drei Studien aber auch eine leicht erhöhte Gefahr an klinisch relevanten Blutungen.

Da sich die Reduktion ischämischer Rezidive insbesondere in den ersten 3-4 Wochen (bei in diesem Zeitraum nur minimal erhöhtem Blutungsrisiko) zeigte, empfehlen nationale wie internationale Leitlinien nun bei Patient ${ }^{*}$ innen mit nicht-kardioembolischem leichten Schlaganfall (NIHSS <4) oder Hochrisiko-TIA (ABCD2-Score $\geq 4$, siehe - Tab. 1) eine Kombinationstherapie mit ASS und Clopidogrel für den ersten Monat, danach Weiterführung einer Monotherapie. Der positive Effekt der dualen Plättchenhemmung dürfte bei makroangiopathischer atherosklerotischer Schlaganfallätiologie am ausgeprägtesten sein.

》) Die Thrombozyten-

aggregationshemmung ist die Basistherapie nach nichtkardioembolischem Schlaganfall

Für die Kombinationstherapie mit ASS und Clopidogrel besteht die stärkste Datenlage (CHANCE- und POINT-Studie), bei Allergie/bekannter fehlender Wirksamkeit von Clopidogrel kann alternativ auch eine Kombinationstherapie von ASS und Ticagrelor (THALES-Studie) verwendet werden und wird in den rezenten DGN-Leitlinien als äquivalent bewertet. Es besteht keine Evidenz für den Nutzen einer längerfristigen dualen Thrombozytenaggregationshemmung (allerdings ist 


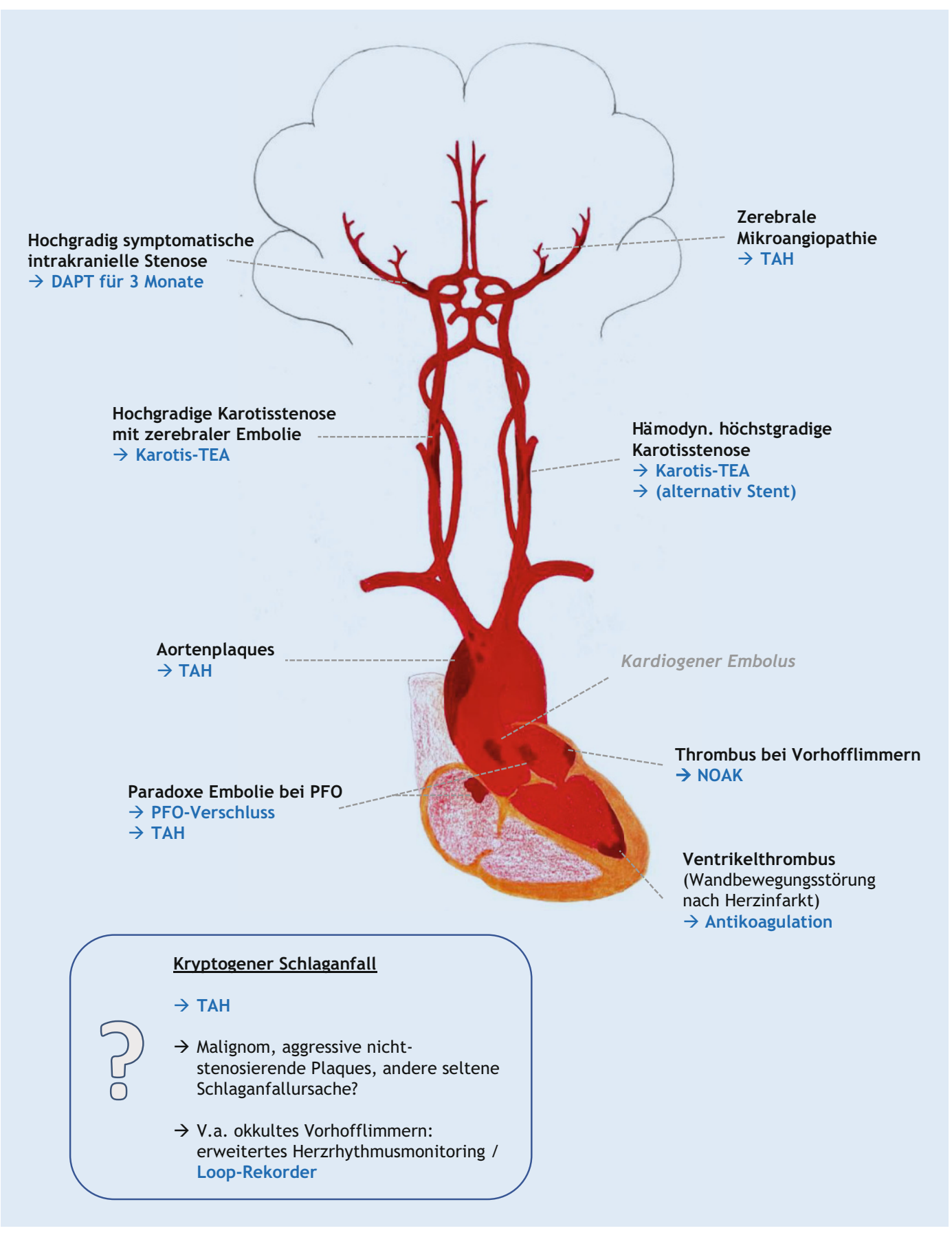

Abb. $1 \triangleleft$ Häufige Schlaganfallätiologien und deren Therapieprinzipien. (TAH Thrombozytenfunktionshemmer, NOAK neue orale Antikoagulantien, $P F O$ persistierendes Foramen ovale, TEA Thrombendarteriektomie, DAPT duale Thrombozytenfunktionshemmung. Copyright: Thomas Gattringer)

bekannt, dass diese mit einem erhöhten Blutungsrisiko einhergeht).

Bei Patient*innen, bei denen keine Kombinationstherapie oder orale Antikoagulation indiziert ist, sollte eine Monotherapie mit ASS erfolgen. Bei Auftreten eines ischämischen Ereignisses unter laufender Therapie mit ASS kann eine Umstellung auf Clopidogrel (oder eventuell Ticagrelor) erwogen werden. Diese Strategie hat sich in der Praxis etabliert, wenngleich hierfür je- doch kaum belastbare Daten vorliegen. Eine große randomisiert-kontrollierte Studie (SOCRATES) verglich ASS mit Ticagrelor in der Sekundärprophylaxe und konnte keine signifikanten Unterschiede der beiden Wirkstoffe hinsichtlich Schlaganfallprävention und Blutungsrisiken zeigen. Der in der akuten Herzinfarkttherapie etablierte Thrombozytenaggregationshemmer Prasugrel ist gemäß Zulassung (vorwiegend aufgrund einer erhöhten Blutungsrate) bei stattge- habtem Schlaganfall einschließlich TIA kontraindiziert.

\section{Orale Antikoagulation}

Hinsichtlich der oralen Antikoagulation haben sich in den letzten Jahren wenige unmittelbare Veränderungen ergeben. Allerdings stand der kryptogene Schlaganfall - d. h. Schlaganfälle, bei denen trotz ausführlicher Abklärung keine sichere Schlaganfallätiologie detektiert werden 
kann - im Vordergrund einiger großer Studien. Die orale Antikoagulation mit NOAK (Rivaroxaban oder Dabigatran) konnte in randomisiert-kontrollierten Studien bei Patient ${ }^{\star}$ innen mit kryptogenem Schlaganfall keinen Vorteil in der Verhinderung von Rezidivschlaganfällen (bei erhöhtem Blutungsrisiko) gegenüber ASS nachweisen. Im Gegensatz steht die adäquate Detektion der paroxysmalen Vorhofflimmerarrhythmie stärker im Fokus: Hier wird bei Patient*innen mit hoher Vortestwahrscheinlichkeit nicht nur auf wiederholte 24-h- und 7-Tage-EKG-Holter zurückgegriffen, sondern zunehmend auch auf die Implantation von Eventrekordern (Loop-Rekorder). Die wichtigsten Prädiktoren zur Detektion einer paroxysmalen Vorhofflimmerarrhythmie sind hohes Alter, Vorhofdilatation, multilokuläre kortikale zerebrale Infarkte (akut und/oder chronisch), reduzierte linksventrikuläre Ejektionsfraktion, erhöhtes NT-proBNP sowie eine hohe Anzahl von supraventrikulären Extrasystolen oder atrialen Runs im Holter-EKG. Ein vorgeschlagener abgestimmter Algorithmus der Österreichischen Schlaganfall Gesellschaft und Kardiologie Gesellschaft zur Detektion einer paroxysmalen Vorhofflimmerarhythmie inkl. Indikation zur Loop-Rekorder-Implantation kann unter diesem Link abgerufen werden: https://www.ögsf.at/wp-content/uploads/2016/11/Positionspapier-

2018_OEGSF_neurologisch.pdf (Positionspapier der Österreichischen Schlaganfall Gesellschaft 2018).

Grundsätzlich sind bei entsprechender Indikation (vorwiegend im Kontext einer Vorhofflimmerarrhythmie) die NOAK (Apixaban, Dabigatran, Edoxaban, Rivaroxaban) den Vitamin-K-Antagonisten (Phenprocoumon, Acenocumarol) aufgrund des geringen Risikos intrazerebraler Blutungen bei zumindest gleichwertiger Risikoreduktion ischämischer Schlaganfälle vorzuziehen. Wichtig ist, NOAK in der korrekten Dosis anzuwenden und eine Dosisreduktion nur dann durchzuführen, wenn diese laut $\mathrm{Zu}$ lassungsbestimmungen indiziert ist (die Verwendung reduzierter Dosierungen in anderen Fällen ist mit einem erhöhten Schlaganfallrisiko verbunden). Der psychopraxis. neuropraxis 2021 - 24:348-355 https://doi.org/10.1007/s00739-021-00758-5

(c) Der/die Autor(en) 2021

\section{S. Fandler-Höfler $\cdot$ M. KneihsI · T. Gattringer}

\section{Update zur Sekundärprophylaxe nach ischämischem Schlaganfall}

\section{Zusammenfassung}

Schlaganfallpatient*innen haben ein substanzielles Risiko, einen Rezidivschlaganfall oder andere vaskuläre Erkrankungen zu erleiden, wobei dieses Risiko vor allem in den ersten Wochen bis Monaten nach dem Schlaganfall signifikant erhöht ist. Neben einer allgemeinen Risikofaktorenkontrolle (Lebensstiländerung inkl. regelmäßiger Bewegung, Verzicht auf Zigarettenrauchen, konsequente Blutdruckeinstellung, LDLCholesterin-Zielwerterreichung, Diabetesmellitus-Einstellung etc.) haben Entwicklungen der letzten Jahre dazu beitragen, das Rezidivrisiko nach Schlaganfall weiter zu senken und Risikopopulationen zu identifizieren, die eine intensivierte Therapie benötigen. Demnach stützt sich eine optimale Sekundärprophylaxe zunehmend auf eine ätiologiebasierte, individuelle Schlaganfallnachsorge und -behandlung. Dieser Artikel liefert einen Überblick über moderne sekundärprophylaktische Therapieprinzipien nach ischämischem Schlaganfall und beleuchtet sowohl medikamentöse als auch interventionelle/operative Strategien.

Schlüsselwörter

Ischämischer Schlaganfall · Rezidivschlaganfall · Sekundärprävention · Ätiologie · Schlaganfallnachsorge

\section{Update on Secondary Prevention After Ischemic Stroke}

\section{Abstract}

Patients with history of stroke have a substantial risk to suffer a recurrent stroke or other major cardiovascular event; this risk is particularly high in the first weeks to months after the index stroke. Besides lifestyle modification and a general control of vascular risk factors, more specific secondary preventive strategies have been identified over the past years. Modern secondary stroke prevention is based on the underlying specific stroke etiology and directed towards an individualized therapeutic strategy and follow-up care. This article aims to provide an overview on recent developments on general as well as more specific preventive measures following an ischemic stroke.

\section{Keywords}

Ischemic stroke $\cdot$ Secondary prevention . Recurrent stroke - Etiology · Poststroke care optimale Zeitpunkt des Starts einer oralen Antikoagulationstherapie mit einem NOAK bei Patient ${ }^{*}$ innen mit Schlaganfall und Vorhofflimmern wird aktuell u. a. in der randomisierten ELAN Studie getestet (kardiales Re-Embolierisiko versus zerebrales Einblutungsrisiko in der Akutphase des Schlaganfalls). Zumeist wird in der Praxis bei Patient*innen mit TIA oder sehr kleinem Schlaganfall in den ersten Tagen mit einem NOAK gestartet, während bei größeren Infarkten 1-2 Wochen zugewartet (und bis dahin mit ASS behandelt) wird.

Wichtige primäre Indikationen für den Einsatz von Vitamin-K-Antagonisten sollten aber im Hinterkopf bleiben (z. B. mechanischer Herzklappenersatz, valvuläres Vorhofflimmern bedingt durch eine hochgradige Mitralklappen- stenose, Antiphospholipid-Antikörpersyndrom).

Bei der wichtigen und häufig spät erkannten Schlaganfallursache einer malignomassoziierten Koagulopathie kommt primär niedermolekulares $\mathrm{He}$ parin in therapeutischer Dosierung oder gemäß neueren Studien alternativ Edoxaban oder Apixaban zum Einsatz. Solche Patient*innen haben ein hohes Risiko für vaskuläre Rezidiverkrankungen (wiederkehrender Schlaganfall, Herzinfarkt, Beinvenenthrombose, Lungenembolie).

\section{Dissektionen}

In der frühen Sekundärprophylaxe nach Dissektionen hirnversorgender Gefäße spielen sowohl ASS als auch Heparin eine Rolle. Randomisierte Studien sowie Metaanalysen belegen keine eindeutigen 
Hier steht eine Anzeige.

\section{曾 Springer}


Tab. 1 ABCD2-Score (Risiko von Rezidivereignissen nach TIA)

\begin{tabular}{l|l}
\hline Risikofaktor & Punkte \\
\hline Alter $\geq 60$ Jahre & 1 \\
\hline $\begin{array}{l}\text { Blutdruck } \geq 140 / 90 \mathrm{~mm} \mathrm{Hg} \\
\text { Klinisch: einseitige Schwäche }\end{array}$ & 1 \\
\hline $\begin{array}{l}\text { Klinisch: Sprachstörung ohne } \\
\text { Schwäche }\end{array}$ & 1 \\
\hline $\begin{array}{l}\text { Dauer der Symptome } \geq 60 \mathrm{~min} \\
\text { Dauer der Symptome } 10-59\end{array}$ & 2 \\
\hline $\begin{array}{l}\text { Diabetes mellitus } \\
\text { Dial }\end{array}$ & 1 \\
\hline
\end{tabular}

Unterschiede hinsichtlich dieser beiden Behandlungsstrategien. Folgende Expertenmeinung findet in der Praxis oftmals Anwendung:

- ASS wird eher angewendet bei größeren Hirninfarkten, intraduraler Dissektion (cave: Risiko einer Subarachnoidalblutung), oder bei ausschließlich lokalen Symptomen durch die Dissektion (z. B. lokale Schmerzen wie Karotidodynie, Horner-Syndrom, kaudale Hirnnervenparese).

- Eine therapeutische Antikoagulation (in der Akutphase mit unfraktioniertem Heparin oder alternativ niedermolekularem Heparin, gefolgt von einer OAK mit Vitamin-KAntagonisten oder gemäß aktueller Fallserien/Expert ${ }^{*}$ innenmeinung mit NOAK) sollte dann erfolgen, wenn multiple, rezidivierende embolische Infarkte trotz TAH-Therapie auftreten, oder eine arterielle (Pseudo-)Okklusion mit erheblicher poststenotischer Flussreduktion ( „low flow“), oder intraluminale arterielle Thromben vorliegen; eventuell auch beim Vorliegen mikroembolischer Signale im transkraniellen Ultraschall.

Die Dauer der Behandlung mittels antithrombotischer/antikoagulatorischer Medikation wird im Einzelfall je nach klinischer Situation (Hirninfarkt vs. nur Lokalsymptome) und dem Verlauf (residuelle Gefäßwandirregularitäten?) individuell festgelegt.

\section{Lipidtherapie}

Der Einsatz lipidsenkender Medikamente ist eine zentrale Säule optimaler Sekundärprophylaxe nach ischämischem Schlaganfall.

Statine sind als medikamentöse FirstLine-Therapie empfohlen. Während frühere Berichte über ein erhöhtes zerebrales Blutungsrisiko unter Statintherapie oder negative Auswirkungen sehr niedriger „low density lipoprotein“ (LDL-)Serumspiegel (u.a. neurokognitive Nebenwirkungen, Diabetes mellitus) in groß angelegten Studien nicht bestätigt wurden, ist die hohe Effektivität der Statintherapie zur Vermeidung zerebrovaskulärer Ereignisse bewiesen (relative Risikoreduktion vaskulärer Rezidivereignisse: 20-30\%).

Nach dem Prinzip „the lower, the better" empfiehlt das aktuelle Positionspapier der Österreichischen Schlaganfallgesellschaft den Einsatz hochpotenter Statine (Atorvastatin, Rosuvastatin) bei allen ischämischen Schlaganfallpatient*innen unabhängig vom Cholesterinstatus (Ausnahme: juveniler Schlaganfall bei Gefäßdissektion etc. ohne Nachweis von Arteriosklerose).

Das LDL ist weiterhin der serologische Ziel-Cholesterin-Marker. Als Mindestziel gilt die $50 \%$ ige LDL-Senkung und das Erreichen eines LDL-Werts $<70 \mathrm{mg} / \mathrm{dl}$. Bei Hochrisikopatient ${ }^{*}$ innen für arterioarteriell embolische Ereignisse (z.B. symptomatische intrakranielle Gefäßstenose, aggressive Karotisplaque, progrediente Arteriosklerose unter Therapie) ist ein LDL-Zielwert von zumindest $<55 \mathrm{mg} / \mathrm{dl}$ anzustreben (ideal $<40 \mathrm{mg} / \mathrm{dl}$ )

\section{》) Eine konsequente LDL- Senkung mit Statinen ist eine zentrale Säule optimaler Sekundärprophylaxe}

Gelingt dieses Vorhaben trotz hochpotenter Statintherapie in maximal verträglicher Dosierung (Atorvastatin $80 \mathrm{mg} /$ Tag; Rosuvastatin $40 \mathrm{mg} / \mathrm{Tag}$ ) nicht, ist der additive Einsatz von Ezetimib (Dosierung: $10 \mathrm{mg} / \mathrm{Tag}$ ) emp- fohlen. Bei schwer/nicht einstellbaren Patient ${ }^{*}$ innen kann der zusätzliche Einsatz der PCSK-9-Inhibitoren Evolocumab (Repatha ${ }^{\circledR}$ ) oder Alirocumab (Praluent ${ }^{\circledR}$ ) über eine spezialisierte Lipidstoffwechselambulanz nötig sein.

Rezent entwickelte therapeutische Alternativen (u.a. Bempedoinsäure, Inclisiran) werden das medikamentöse Portfolio künftig noch ergänzen.

Wichtig in jedem Fall ist die regelmäßige Kontrolle der Lipidstoffwechselparameter zur Überprüfung der Therapieadhärenz/der LDL-Zielwerte.

\section{Operative/interventionelle Verfahren}

\section{Thrombendarteriektomie/Stenting}

Neben der medikamentösen Therapie ist die rasche und zielgerichtete Indikationsstellung operativer Verfahren essenziell für die Prognose ischämischer Schlaganfallpatient*innen.

Dies gilt insbesondere für die symptomatische extrakranielle A.-carotis-interna-Stenose: Die aktuellen Guidelines der European Stroke Organisation unterstreichen die Wertigkeit der Karotisthrombendarteriektomie (TEA) zur Behandlung symptomatischer 50-99\%iger (NASCET-Klassifikation) Karotisstenosen. Im Vergleich zum Karotisstenting ist die TEA insbesondere ab dem 70. Lebensjahr (geringeres periprozedurales Schlaganfallrisiko) und bei moderatem Stenoseausmaß $50-70 \%$ zu bevorzugen. Bei jüngeren Patient*innen oder besonderen klinischen Konstellationen (z.B. radiogene Stenose nach Bestrahlung im Hals-Kopf-Bereich, voroperierte Stenose) stellt das endovaskuläre Stentverfahren eine wichtige Alternative dar.

Unabhängig vom Verfahren wird die frühe Sanierung der symptomatischen Karotisstenose innerhalb von spätestens 2 Wochen nach dem Ereignis empfohlen, wenngleich v.a. bei TIA und kleinen Hirninfarkten bzw. bei Patient ${ }^{*}$ innen mit Hochrisikostenose (rezidivierende Schlaganfallsymptomatik) eine Karotisoperation in den ersten Tagen zu bevorzugen ist. Die Morphe des Hirninfarkts (u.a. Größe, Einblutung), der klinische Zustand der 
Patient ${ }^{*}$ innen und die Wahrscheinlichkeit für perioperative Komplikationen fließen in die Wahl des OP-Termins ein. Nach erfolgter Karotisrevaskularisation ist ein strenges Blutdruckmanagement (zur Vermeidung eines zerebralen Hyperperfusionssyndroms) sowie generell ein engmaschiges vaskuläres Risikofaktorenmanagement (inkl. Einsatz von Thrombozytenfunktionshemmern und Statinen) zur Vermeidung einer Rezidivstenose sowie weiterer vaskulärer Ereignisse (Schlaganfall, Herzinfarkt, PAVK) essenziell. Generell sollten alle Schlaganfallpatient*innen, speziell aber diejenigen mit Makroangiopathien der hirnversorgenden Gefäße auf das Vorliegen einer Claudicatio intermittens (PAVK), Niereninsuffizienz bzw. koronaren Herzerkrankung gescreent werden.

Die interdisziplinäre Falldiskussion von Neurolog*innen, Gefäßchirurg*innen und interventionellen $\mathrm{Ra}$ diolog*innen ist für die Findung der optimalen individuellen Therapie ent- scheidend. Dies gilt insbesondere auch für symptomatische und morphologisch aggressive Karotisstenosen $<50 \%$, die trotz maximaler konservativ-medikamentöser Therapie zu einem Rezidivereignis geführt haben (• Abb. 2).

Bei symptomatischen Stenosen der A. vertebralis sowie intrakraniellen Stenosen (z. B. der A. cerebri media oder basilaris) ist der Stellenwert der Stentbehandlung aktuell gering und sollte nur in seltenen Einzelfällen erwogen werden. So gilt primär ein „best medical treatment" (Thrombozytenfunktionshemmer, hochpotentes Statin etc.) als Therapie der Wahl. Nur bei Rezidivereignissen unter maximaler medikamentöser Therapie sind Stentbehandlungen zu erwägen. Seit Publikation der SAMMPRISStudie im Jahr 2011, einer vorzeitig abgebrochenen Studie, die zeigen konnte, dass ein intensiviertes medikamentöses Therapieregime einer intrakraniellen Stentbehandlung deutlich überlegen ist, besteht die Therapie der Wahl der hoch- gradigen symptomatischen intrakraniellen arteriellen Stenose aus einer dualen Thrombozytenfunktionshemmung mit ASS und Clopidogrel für 3 Monate, einem hochpotenten Statin sowie einer konsequenten Blutdruckeinstellung, einer Nikotinkarenz sowie Gewichtsreduktion. Auch eine kürzlich publizierte populationsbasierte Studie aus Oxford, UK, die im Gegensatz zu SAMMPRIS auch ältere Patient ${ }^{*}$ innen ( $>60$ Jahre) untersuchte, bestätigte die Effektivität dieser Therapiestrategie zur Schlaganfallsekundärprophylaxe bei hochgradigen symptomatischen intrakraniellen Stenosen.

Bei symptomatischen Aortenplaques, die $\mathrm{zu}$ einer arterio-arteriellen zerebralen Embolie geführt haben, ist ebenso ein intensiviertes medikamentöses Therapieregime bestehend aus Thrombozytenfunktionshemmern und hochpotenten Statinen etc. angezeigt.

Hier steht eine Anzeige. 

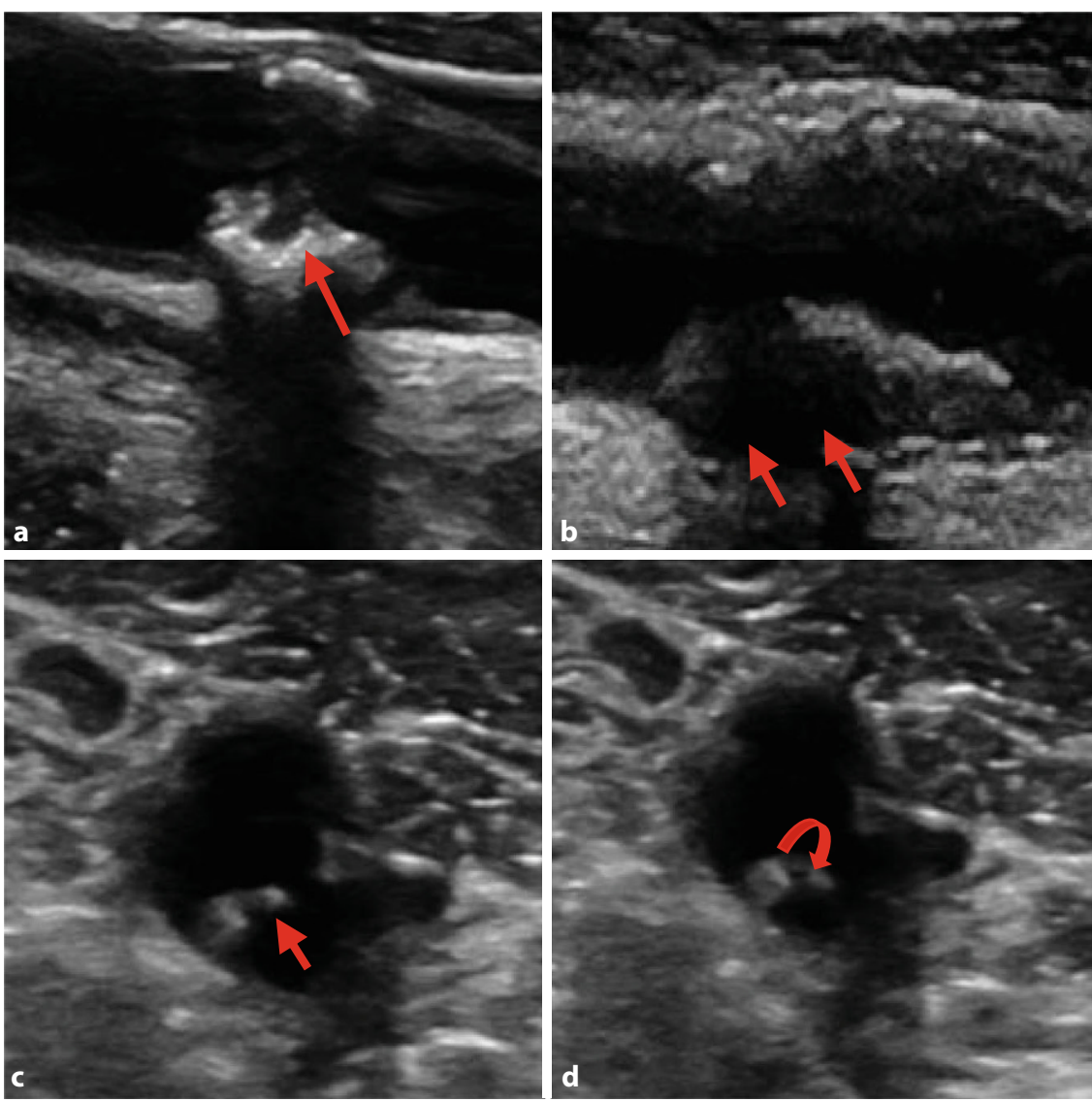

Abb. $2 \Delta$ Features von Karotisplaques mit erhöhtem arterio-arteriellen embolischen Risiko. (Copyright:Universitätsklinik für Neurologie Graz). Sonographische Darstellung von exulzerierten (a), hypoanechogenen (b) und mobilen ( $\mathbf{c}, \mathbf{d}$ ) Gefäßwandablagerungen als typische Zeichen für das Vorliegen einer Hochrisikoplaque

\section{Persistierendes Foramen ovale (PFO)}

Die paradoxe zerebrale Embolie bei PFO und funktionellem Rechts-Links-Shunt ist eine zu beachtende Ätiologie des juvenilen Schlaganfalls. Aufgrund der hohen PFO-Prävalenz in der Bevölkerung ist die Unterscheidung zwischen Koinzidenz und wahrscheinlicher Schlaganfallursache immanent.

Große kontrolliert-randomisierte Studien (RESPECT, CLOSE, GORE REDUCE) lassen auf einen geringen bis moderaten sekundärprophylaktischen Vorteil eines PFO-Verschlusses bei ausgewählten jungen Schlaganfallpatient*innen (<60. Lebensjahr) ohne konkurrierende Schlaganfallätiologie/ohne vaskuläre Risikofaktoren und Vorliegen eines funktionell relevanten RechtsLinks-Shunt schließen.
Besonders scheinen Patient ${ }^{*}$ innen mit einem assoziierten Vorhofseptumaneurysma von einer PFO-Okklusion zu profitieren. Der Risk-of-Paradoxical-Embolism-(ROPE-)Score zur Abschätzung der Rolle des PFOs bei kryptogenem Schlaganfall hat sich in der Praxis in zahlreichen aktuellen Studien als hilfreich in der Selektion zum PFO-Verschluss erwiesen. Generell gilt, dass ein Thrombozytenfunktionshemmer unabhängig vom PFO-Verschluss aus Sicht der meisten Schlaganfallexpert ${ }^{*}$ innen lebenslang indiziert ist. Da PFO-assoziierte Rezidivschlaganfälle insgesamt selten sind, ist es essenziell, bei diesen Patient ${ }^{*}$ innen eine genaue Re-Evaluation der Schlaganfallursache einzuleiten.

\section{Herzohrverschluss}

Der therapeutische Herzohrverschluss (z.B. Watchman ${ }^{\circledR}$ Device, Boston Scien- tific, St. Paul, MN, USA) bei kardiovaskulären Risikopatient*innen mit Vorhofflimmern und hohem (Hirn-)Blutungsrisiko ist durch die Entwicklung der NOAKs mit besserem Sicherheitsprofil im Vergleich zu Warfarin in den Hintergrund gerückt. Dennoch können Sondersituationen (z.B. Patient*innen mit Vorhofflimmern mit stark erhöhtem Hirnblutungsrisiko wie etwa beim Vorliegen einer schweren zerebralen Amyloidangiopathie oder einem Z.n. mehreren Blutungsereignissen) vorliegen, die im Einzelfall den Einsatz eines Herzohrverschlusses sinnvoll erscheinen lassen. Die eingehende Falldiskussion unter Einbeziehung des klinischen Patient ${ }^{*}$ innenzustands und möglicher therapeutischer Alternativen im interdisziplinären Setting (Neurologie und Kardiologie) ist jedenfalls zu empfehlen.

\section{Post Stroke Disease Management}

Während bei anderen Erkrankungen wie Krebs oder nach Herzinfarkten standardisierte Nachsorgeprogramme etabliert sind, gab es bis vor Kurzem wenig Daten aus dem Schlaganfallbereich. Die rezent publizierte randomisierte StrokeCard-Studie aus Österreich (Med Uni Innsbruck, Barmherzige Brüder Wien) konnte nachweisen, dass ein pragmatisches, strukturiertes Nachsorgekonzept zu einer $>30 \%$ igen Reduktion von vaskulären Rezidiverkrankungen (Schlaganfall, Herzinfarkt, vaskulärer Tod) im ersten Jahr nach Schlaganfall/TIA führen kann. So zeigten 5,4\% der Patient ${ }^{*}$ innen, die eine strukturierte, multiprofessionelle ambulante Nachsorge 3 Monate nach dem zerebrovaskulären Ereignis (Kontrolle der neurologischen Funktion und der Rehabilitationsziele, Erfassung und Kontrolle der vaskulären Risikofaktoren sowie von schlaganfallassoziierten Komplikationen durch Neurolog*innen, Therapeut ${ }^{*}$ innen, Pflegepersonen) erhielten, ein vaskuläres Rezidiv nach einem Jahr, während dies bei $8,3 \%$ in der Kontrollgruppe der Fall war. Zudem war die Lebensqualität und der funktionelle neurologische Zustand der Schlaganfallpatient ${ }^{*}$ innen in der Interventionsgruppe verbessert. Derartige ambulante 
Post-Stroke-Disease-Management-Programme könnten künftig durch flächendeckendes Angebot zu einer optimierten multiprofessionellen vaskulären Schlaganfallsekundärprävention maßgeblich beitragen.

\section{Fazit für die Praxis}

- Eine konsequente Einstellung der modifizierbaren kardiovaskulären Risikofaktoren stellt das Rückgrat der Schlaganfallsekundärprophylaxe dar.

- Die konkrete Schlaganfallätiologie determiniert die spezifische Schlaganfallsekundärprophylaxe.

- Thrombozytenfunktionshemmer bleiben die Standardtherapie nach nicht-kardioembolischem (inklusive kryptogenem) Schlaganfall.

- Bei selektierten Patient*innen mit Hochrisiko-TIA/,"minor stroke" senkt eine vorübergehende duale Thrombozytenfunktionshemmertherapie (ASS + Clopidogrel oder ASS + Ticagrelor) für 1 Monat das ischämische Rezidivrisiko bei leicht erhöhtem Blutungsrisiko.

- NOAK sind die Therapie der Wahl bei Patient*innen mit Vorhofflimmerarrhythmie-assoziiertem Schlaganfall.

- Nach ischämischem Schlaganfall sollte bis auf wenige Ausnahmen zumindest ein LDL-Zielwert $<70 \mathrm{mg} / \mathrm{dl}$ (bei Hochrisikopatient*innen $<55 \mathrm{mg} / \mathrm{dl}$ ) durch Einsatz hochpotenter Statine, Ezetimib oder PCSK-9-Inhibitoren erreicht werden.

- Bei symptomatischen höhergradigen A.-carotis-interna-Abgangsstenosen ist die chirurgische Thrombendarteriektomie innerhalb von 2 Wochen nach dem Schlaganfall die Therapie der Wahl und prinzipiell dem endovaskulären Stenting vorzuziehen.

- Neuere Studien konnten einen moderaten Vorteil des katheterinterventionellen PFO-Verschlusses bei ausgewählten Schlaganfallpatienten/-innen ( $<60$ Jahre, relevanter Rechts-Links-Shunt, assoziiertes Vorhofseptumaneurysma, keine konkurrierende Ätiologie) nachweisen.

- Eine interdisziplinäre, standardisierte Nachbehandlung von Schlaganfallpatient*innen an einem neurologi- schen Expert*innenzentrum kann die Prognose verbessern und sollte künftig flächendeckend angeboten werden.

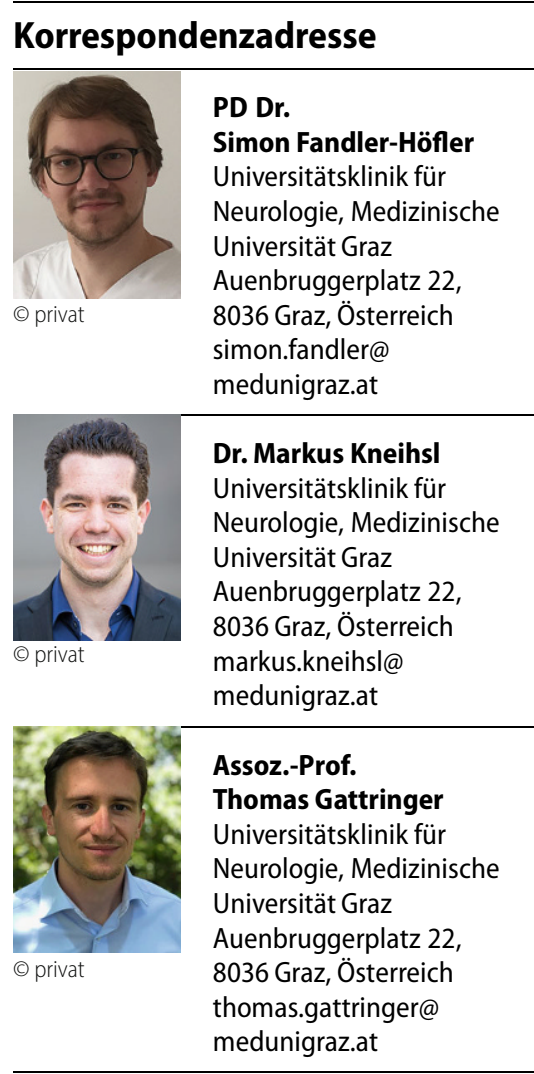

Funding. Open access funding provided by Medical University of Graz.

\section{Einhaltung ethischer Richtlinien}

Interessenkonflikt. S. Fandler-Höfler, M. Kneihsl und T. Gattringer geben an, dass kein Interessenkonflikt besteht.

Für diesen Beitrag wurden von den Autoren keine Studien an Menschen oder Tieren durchgeführt. Für die aufgeführten Studien gelten die jeweils dort angegebenen ethischen Richtlinien.

Open Access. Dieser Artikel wird unter der Creative Commons Namensnennung 4.0 International Lizenz veröffentlicht, welche die Nutzung, Vervielfältigung Bearbeitung, Verbreitung und Wiedergabe in jeglichem Medium und Format erlaubt, sofern Sie den/die ursprünglichen Autor(en) und die Quelle ordnungsgemäß nennen, einen Link zur Creative Commons Lizenz beifügen und angeben, ob Änderungen vorgenommen wurden.

Die in diesem Artikel enthaltenen Bilder und sonstiges Drittmaterial unterliegen ebenfalls der genannten Creative Commons Lizenz, sofern sich aus der Abbildungslegende nichts anderes ergibt. Sofern das betreffende Material nicht unter der genannten Creative
Commons Lizenz steht und die betreffende Handlung nicht nach gesetzlichen Vorschriften erlaubt ist, ist für die oben aufgeführten Weiterverwendungen des Materials die Einwilligung des jeweiligen Rechteinhabers einzuholen.

Weitere Details zur Lizenz entnehmen Sie bitte der Lizenzinformation auf http://creativecommons.org/ licenses/by/4.0/deed.de.

Hinweis des Verlags. Der Verlag bleibt in Hinblick auf geografische Zuordnungen und Gebietsbezeichnungen in veröffentlichten Karten und Institutsadressen neutral. 